\title{
Probing the proton and its excitations in full QCD
}

\author{
Benjamin Owen ${ }^{* i a}$, Waseem Kamleh ${ }^{a}$, Derek Leinweber ${ }^{a}$, Selim Mahbub ${ }^{a, b}$ and \\ Benjamin Menadue ${ }^{a, c}$
}

${ }^{a}$ Special Research Centre for the Subatomic Structure of Matter (CSSM),

School of Chemistry and Physics, University of Adelaide, SA 5005, Australia

${ }^{b}$ CSIRO Computational Informatics, College Road, Sandy Bay, TAS 7005, Australia

${ }^{c}$ National Computational Infrastructure,

Australian National University, Canberra, ACT 0200, Australia

E-mail: benjamin.owendadelaide.edu.au

We present a first look at the application of variational techniques for the extraction of the electromagnetic properties of an excited nucleon system. In particular, we include preliminary results for charge radii and magnetic moments of the proton, its first even-parity excitation and the $\Delta^{+}$.

31st International Symposium on Lattice Field Theory LATTICE 2013

July 29 - August 3, 2013

Mainz, Germany

* Speaker.

${ }^{\dagger}$ We thank PACS-CS Collaboration for making their $2+1$ flavor configurations available and acknowledge the important ongoing support of the ILDG. This research was undertaken with the assistance of resources at the NCI National Facility in Canberra, Australia, and the iVEC facilities at Murdoch University (iVEC@Murdoch) and the University of Western Australia (iVEC@UWA). These resources were provided through the National Computational Merit Allocation Scheme and the University of Adelaide Partner Share which are supported by the Australian Government. We also acknowledge eResearch SA for their support of our supercomputers. This research is supported by the Australian Research Council. 


\section{Introduction}

In our recent work [四] we performed a calculation of $g_{A}$ using the variational method. We found that this provides an automatic approach for suppressing excited state effects, eliminating the need for fine-tuning.

Isolation of the eigenstates results in rapid ground state dominance. This allows an earlier current insertion in three-point functions. Fit windows commence earlier and are wider, suppressing excited-state systematic error and reducing statistical uncertainties. Similar findings were presented for the rho meson in Ref. [目].

The use of the variational approach has the added benefit of providing access to excited state matrix elements. This is the motivation of a growing number of studies that are moving beyond the lowest energy eigenstates to better understand the dynamics of QCD. Here we apply the correlation matrix to calculate the electromagnetic form factors of the ground-state proton, its first even-parity excitation and the $\Delta^{+}$. From these we extract the charge radius and magnetic moments for these states. To the best of our knowledge, this is the first determination of the electromagnetic form factors of the first even-parity excitation of the nucleon.

\section{Correlation matrix methods for matrix elements}

The goal of this approach is to produce a set of operators $\phi^{\alpha}$ that satisfy

$$
\left\langle\Omega\left|\phi^{\alpha}\right| \beta, p, s\right\rangle=\delta^{\alpha \beta} .
$$

The way in which this is achieved is to take an existing basis of operators $\left\{\chi_{i}\right\}$ and construct the operators $\phi^{\alpha}$ as a linear superposition

$$
\phi^{\alpha}(x)=\sum_{i} v_{i}^{\alpha} \chi_{i}(x), \quad \bar{\phi}^{\alpha}(x)=\sum_{j} \bar{\chi}_{j}(x) u_{j}^{\alpha} .
$$

Beginning with the matrix of cross correlators

$$
G_{i j}(\vec{p}, t)=\sum_{\vec{x}} e^{-i \vec{p} \cdot \vec{x}}\left\langle\Omega\left|\chi_{i}(x) \bar{\chi}_{j}(0)\right| \Omega\right\rangle,
$$

and noting $G_{i j}(\vec{p}, t) u_{j}^{\alpha}$ provides a recurrence relation with time dependence $e^{-E_{\alpha} t}$, one can show, as demonstrated in [B]], that the necessary vectors $v_{i}^{\alpha}$ and $u_{j}^{\alpha}$ are the eigenvectors of the generalised eigenvalue equations

$$
\begin{aligned}
v_{i}^{\alpha} G_{i j}\left(\vec{p}, t_{0}+\Delta t\right) & =e^{-E_{\alpha} \Delta t} v_{i}^{\alpha} G_{i j}\left(\vec{p}, t_{0}\right), \\
G_{i j}\left(\vec{p}, t_{0}+\Delta t\right) u_{j}^{\alpha} & =e^{-E_{\alpha} \Delta t} \quad G_{i j}\left(\vec{p}, t_{0}\right) u_{j}^{\alpha} .
\end{aligned}
$$

It is worth noting that these equations are evaluated for a given 3-momentum $\vec{p}$ and so the corresponding operators satisfy Eq. (L. $($ d) for this momentum only. One can project out the correlator for the state $\alpha$ by

$$
G^{\alpha}(\vec{p}, t) \equiv v_{i}^{\alpha}(\vec{p}) G_{i j}(\vec{p}, t) u_{j}^{\alpha}(\vec{p})
$$


from which the desired quantities are extracted in the standard way. To access the corresponding three-point correlator, it is a simple matter of applying the relevant eigenvectors to the corresponding three-point function, where care is taken to ensure that the projection is done with the correct momenta for source and sink:

$$
G^{\alpha}\left(\vec{p}^{\prime}, \vec{p}, t_{2}, t_{1}\right) \equiv v_{i}^{\alpha}\left(\vec{p}^{\prime}\right) G_{i j}\left(\vec{p}^{\prime}, \vec{p}, t_{2}, t_{1}\right) u_{j}^{\alpha}(\vec{p}) .
$$

From the projected two and three-point functions one then constructs a suitable ratio in the standard way to isolate the desired matrix element. Here we choose to use the ratio as defined in [团]

$$
R^{\alpha}\left(\vec{p}^{\prime}, \vec{p} ; \Gamma\right)=\sqrt{\frac{G^{\alpha}\left(\vec{p}^{\prime}, \vec{p}, t_{2}, t_{1} ; \Gamma, \Gamma_{4}\right) G^{\alpha}\left(\vec{p}, \vec{p}^{\prime}, t_{2}, t_{1} ; \Gamma, \Gamma_{4}\right)}{G^{\alpha}\left(\vec{p}^{\prime}, t_{2} ; \Gamma_{4}\right) G^{\alpha}\left(\vec{p}, t_{2} ; \Gamma_{4}\right)}} .
$$

To isolate the electric and magnetic form factors, we follow the approach outlined in [\$] for the proton, and [G] for the $\Delta^{+}$baryon. In the following, we have projected out the relevant correlators and constructed the relevant ratios for the state $|\alpha\rangle$ and so will drop the state label $\left(R \equiv R^{\alpha}\right)$. We also define the reduced ratio $\bar{R}$ as

$$
\bar{R}^{\mu}\left(\vec{p}^{\prime}, \vec{p} ; \Gamma\right)=\left[\frac{2 E_{p}}{E_{p}+M}\right]^{1 / 2}\left[\frac{2 E_{p^{\prime}}}{E_{p^{\prime}}+M}\right]^{1 / 2} R^{\mu}\left(\vec{p}^{\prime}, \vec{p} ; \Gamma\right) .
$$

\section{Baryon Form Factors}

The electromagnetic interaction with a spin- $-1 / 2$ system is described by two independent form factors. As we are interested in the static properties of these hadrons, we shall consider the Sachs form factors $G_{E}$ (charge) and $G_{M}$ (magnetic). We choose the incoming state to be at rest and so as outlined in Refs. [[], 四] we can extract $G_{E}$ and $G_{M}$ through the following ratio terms:

$$
G_{E}\left(q^{2}\right)=\bar{R}^{4}\left(\vec{q}, 0 ; \Gamma_{4}\right), \quad G_{M}\left(q^{2}\right)=\frac{(E+M)}{\left|\vec{q}_{1}\right|} \bar{R}^{3}\left(\vec{q}, 0 ; \Gamma_{2}\right) .
$$

The electromagnetic interaction with a spin-3/2 system is described by four independent form factors. Again we consider the Sachs form factors which are $G_{E 0}$ (charge), $G_{M 1}$ (magnetic-dipole), $G_{E 2}$ (electric-quadrupole) and $G_{M 3}$ (magnetic-octupole). In this work will evaluate the charge radii and magnetic moments only. Again, we choose the incoming state to be at rest and so as outlined in [目] we can extract $G_{E 0}$ and $G_{M 1}$ through the following ratio terms:

$$
\begin{aligned}
G_{E 0} & =\frac{1}{3}\left(\bar{R}_{1}{ }^{4}{ }_{1}\left(\vec{q}, 0 ; \Gamma_{4}\right)+\bar{R}_{2}{ }^{4}{ }_{2}\left(\vec{q}, 0 ; \Gamma_{4}\right)+\bar{R}_{3}{ }_{3}{ }_{3}\left(\vec{q}, 0 ; \Gamma_{4}\right)\right), \\
G_{M 1} & =-\frac{3}{5} \frac{E+M}{\left|\vec{q}_{1}\right|}\left(\bar{R}_{1}{ }^{3}{ }_{1}\left(\vec{q}, 0 ; \Gamma_{2}\right)+\bar{R}_{2}{ }^{3}{ }_{2}\left(\vec{q}, 0 ; \Gamma_{2}\right)+\bar{R}_{3}{ }^{3}{ }_{3}\left(\vec{q}, 0 ; \Gamma_{2}\right)\right) .
\end{aligned}
$$

\subsection{Charge Radii and Magnetic Moments}

To extract the squared charge radius, we assume a dipole Ansatz. For the magnetic moments, we assume that the charge and magnetic form factors display common scaling at low $Q^{2}$ such that $G_{M}(0)=G_{M}\left(Q^{2}\right) / G_{E}\left(Q^{2}\right)$ for a unit charge baryon and the magnetic moment is

$$
\mu_{B}=G_{M}(0) \frac{e}{2 M_{B}}=G_{M}(0) \frac{M_{N}}{M_{B}} \frac{e}{2 M_{N}}=G_{M}(0) \frac{M_{N}}{M_{B}} \mu_{N}
$$

where we choose to express the moments in nuclear magnetons, $\mu_{N}$. 


\section{Calculation Details}

The key to success with the variational approach is to utilise a basis of operators which provide a large span within excited state spectrum. As there are a limited number of local operators for a given $J^{P C}$, a great deal of work has been made by various groups in increasing the available operators. Here we choose to use gauge-invariant Gaussian smearing of the fermion source and sinks as a method of extending our operator basis [ $[\mathbb{0}$, Q $]$ ]. We choose to use $16,35,100$ and 200 sweeps of smearing in the spatial dimensions with smearing fraction $\alpha=0.7$ [ [8] allowing for the construction of a $4 \times 4$ correlation matrix. The proton and $\Delta^{+}$are accessed with the standard interpolators

$$
\chi(x)=\varepsilon^{a b c}\left(u^{T a}(x) C \gamma_{5} d^{b}(x)\right) u^{c}(x), \quad \text { and } \quad \chi_{i}(x)=\varepsilon^{a b c}\left(u^{T a}(x) C \gamma_{i} d^{b}(x)\right) u^{c}(x),
$$

respectively. For the variational analysis we use $t_{0}=18$ and $\Delta t=2$ relative to the source at $t=16$.

The ensembles used in this calculation are the PACS-CS $2+1$ flavour dynamical-QCD gauge-

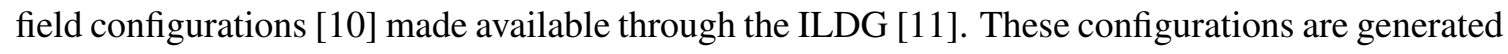
using an $\mathscr{O}(a)$-improved Wilson-Clover fermion action and Iwasaki gauge-action, with $\beta=1.90$ resulting in a lattice spacing $a=0.0907 \mathrm{fm}$. The lattices have dimension $32^{3} \times 64$ giving rise to a spatial box of length $L=2.9 \mathrm{fm}$. We consider four masses for the ground state proton and $\Delta^{+}$and the heaviest two for the proton excitation. For each mass, we have 350 configurations.

The quark source is at $t=16$ relative to a fixed boundary condition defining $t=1$. For the SST inversion we choose to use the fixed current method with a conversed-vector current inserted at $t_{S}=21$. For our error analysis we use a second-order single-elimination jackknife method. The $\chi^{2}$ per degree of freedom, $\chi_{\mathrm{dof}}^{2}$, is obtained via a covariance matrix analysis.

\section{Results}

To ensure that we are in fact probing a nucleon excitation throughout the Euclidean time range required, we consider the behaviour of the projected two-point correlator. In Fig. 1 we display $\log (G)$ for the first excited state of the proton from the variational analysis. The linear behaviour observed between times $t=20-26$ indicates that we have successfully isolated a single eigenstate by $t=20$ (prior to the current insertion at $t_{S}=21$ ) and that this eigenstate dominates the correlator over the region considered in calculating the form factors.

Our results for the electric, $G_{E}$, and magnetic, $G_{M}$, form factors for the first even-parity excitation of the proton, $p^{\prime}$, are displayed in Fig. 2 where we can easily identify plateaus in both cases. Noting that the terms contributing to this result are usually hidden by the dominant ground state within a standard unprojected correlator, the ability to extract a result and for the duration which we observe highlights the enormous power that the variational method offers.

Fig. 3 illustrates our results for the squared charge radii and magnetic moments of the proton, $\Delta^{+}$and the first even-parity excitation of the proton, $p^{\prime}$. There are three key observations that we will highlight in the following.

Firstly, the proton and $\Delta^{+}$baryon have very similar form factors across the four masses considered. In Refs. [12] for example, it was shown that this behaviour is expected for the magnetic moment where cancellations of the $\Delta^{++}-\pi^{-}$and $n-\pi^{+}$loop corrections to the $\Delta^{+}$leave a similar 


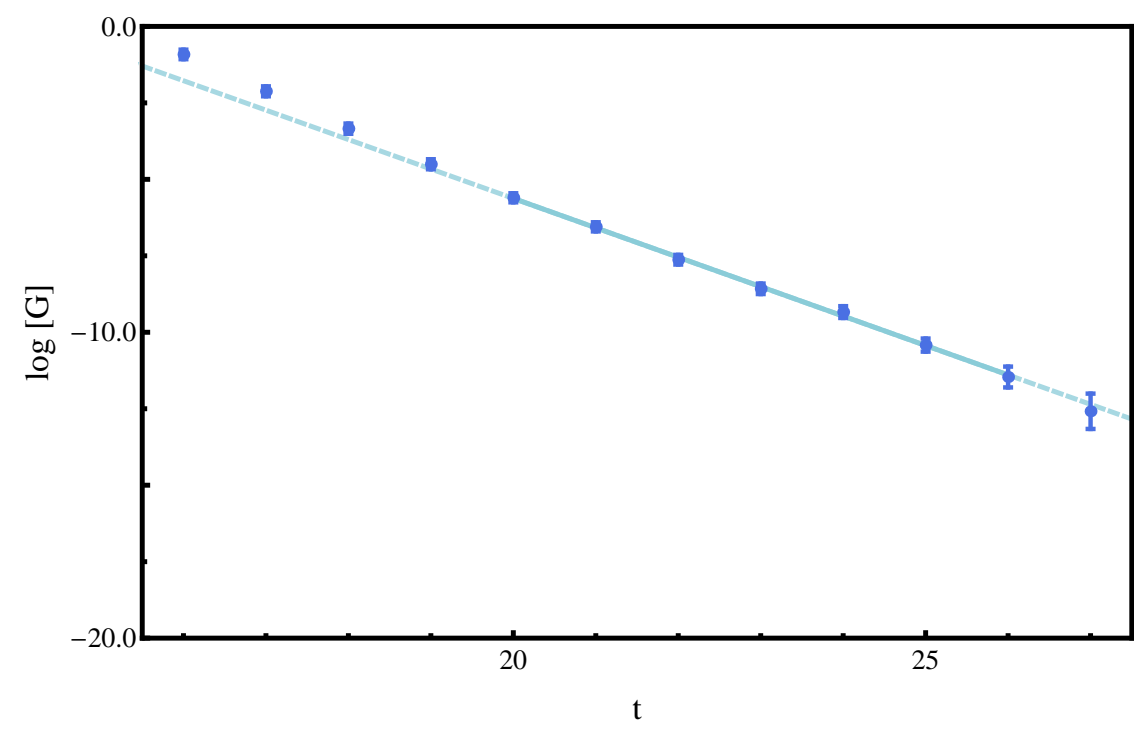

Figure 1: $\log (G)$ for the projected correlator for the first even-parity excitation of the nucleon. The solid line is the best fit line highlighting the linear behaviour of the correlator commencing at $t=20$ prior to the current insertion. The correlator is consistent with a single isolated excited state over the region where the form factors will be analysed.
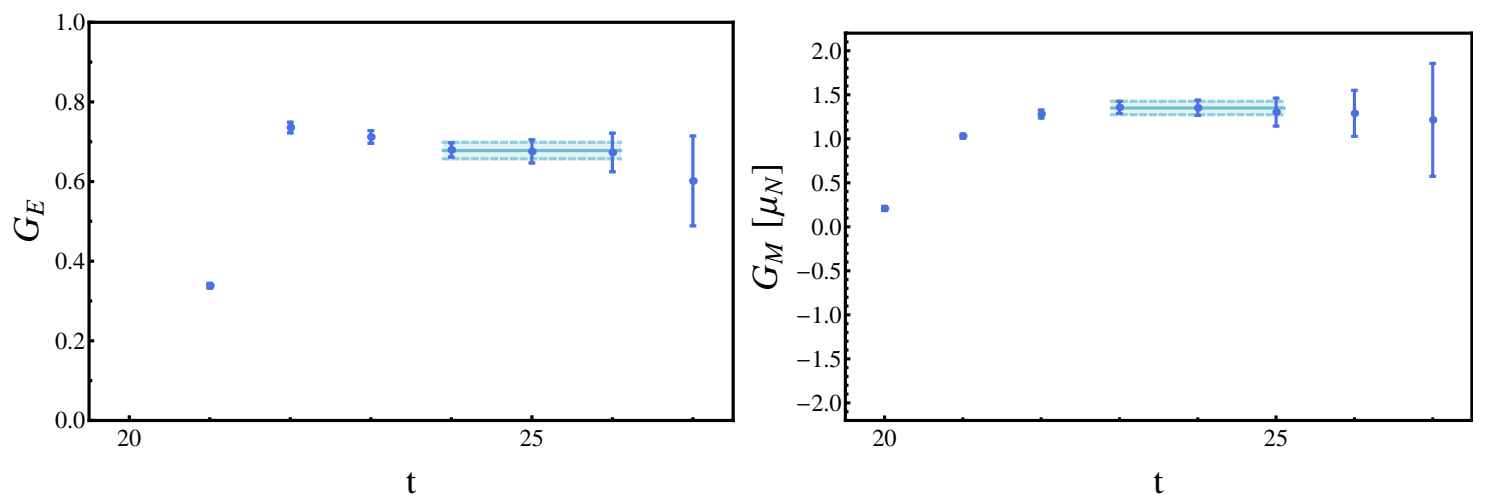

Figure 2: Ratios of two- and three-point correlation functions providing the electromagnetic form factors of the first even-parity excitation of the proton at $Q^{2}=0.146 \mathrm{GeV}^{2}$. The electric form factor, $G_{E}$, (left) and the magnetic form factor, $G_{M}$, (right) are illustrated. Plateaus commence as early as two time slices following the current insertion at $t_{S}=21$.

chiral behaviour for the proton and $\Delta^{+}$. Our result invites further investigation near the physical point where the opening of the $N \pi$ threshold will change the interplay between these contributions.

The second key observation is that the first even-parity excitation of the proton is significantly larger than the ground state. This is consistent with the identification of this state being a radial excitation of the proton through an examination of the three-quark wave function of this state [ए3]]. However, it should also be noted that at the two values of $m_{\pi}^{2}$ considered herein, the energies of these first even-parity excitations of the nucleon sit close to the $N \pi P$-wave scattering threshold. One cannot rule out that we may be probing an eigenstate dominated by a two-particle $N \pi$ scattering 

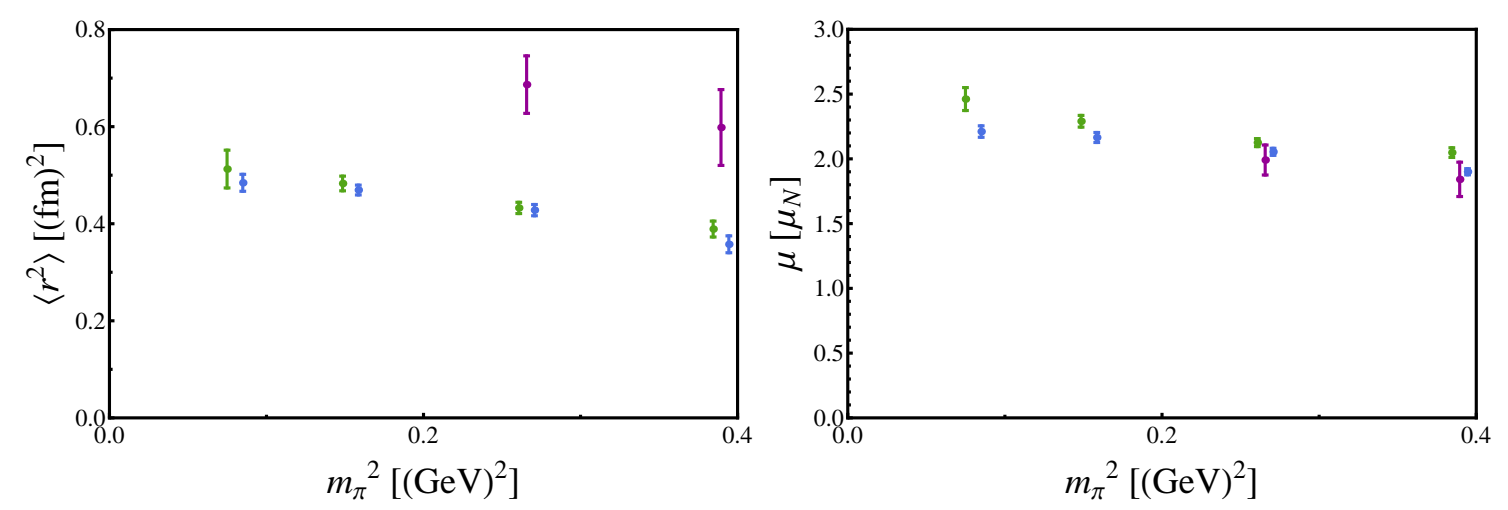

Figure 3: Left: The squared charge radius, $\left\langle r_{E}^{2}\right\rangle$, for the proton (blue), $\Delta^{+}$(green) and the first even-parity excitation of the proton, $p^{\prime}$ (purple) in units of (fm) $)^{2}$. Right: The magnetic moment, $\mu$, for the proton (blue), $\Delta^{+}$(green) and the first even-parity excitation of the proton, $p^{\prime}$ (purple) in units of $\mu_{N}$. The blue and green data points have been offset symmetrically for clarity.

component. As the $N \pi$ interaction is attractive and our box volume is $2.9 \mathrm{fm}$, the RMS-diameter of $\sim 1.7 \mathrm{fm}$ seen at the lighter of the two ensembles considered may be reflecting an underlying multi-particle structure.

Perhaps the most interesting observation is that the magnetic moment of the first even-parity excitation, $p^{\prime}$, is similar to that of the proton and $\Delta^{+}$. While the most naive quark models would draw on the $2 S$ nature of the state and predict a magnetic moment equal to the ground state magnetic moment of the proton, one might have anticipated interesting effects associated with $P$-wave multiparticle orbital angular momentum contributions to this state.

One might also expect effects associated with the increased mass of the excited state. Referring to Eq. (B.2), we note that the magnetic moment is suppressed by the hadron mass which appears in the natural magneton of the moment. However this effect has been compensated by changes in the $Q^{2}$ dependence of the form factor between the ground and excited state reflected in the electric form factors.

Thus, the predictions of the naive constituent quark model in the charge-symmetric limit, $\mu_{p}=\mu_{\Delta^{+}}=\mu_{p^{\prime}}$, have emerged from the complexities of quantum field theory. As predicted in the AccessQM model of Ref. [14] one will have to wait for simulations in the light quark-mass regime of QCD to see its rich structure.

\section{Conclusion}

In this work we have demonstrated the utility of the variational method in calculating the electromagnetic form factors of excited states of the nucleon. It enables a robust isolation of the first even-parity excitation of the nucleon over a wide range of Euclidean times and makes it possible to extract the electromagnetic form factors. To the best of our knowledge, this is the first determination of the electromagnetic form factors of the first even-parity excited state of the nucleon.

Comparison with the ground state proton and $\Delta^{+}$baryons highlights the extended nature of this excitation. At the same time, the predictions of early simple constituent quarks models for baryon magnetic moments are seen to emerge from the complex interactions of QCD. Furthermore, we 
observed striking similarities between the proton and $\Delta^{+}$properties as predicted in the AccessQM model [14]].

Extending this work to lighter masses near the $\Delta$ decay threshold holds the promise of exposing interesting dynamics. It will also be interesting to explore the electromagnetic structure of oddparity excitations of the nucleon [15]].

\section{References}

[1] B. J. Owen, J. Dragos, W. Kamleh, D. B. Leinweber, M. S. Mahbub, B. J. Menadue and J. M. Zanotti, Variational Approach to the Calculation of gA, Phys. Lett. B 723, 217 (2013) [arXiv:1212.4668 [hep-lat]].

[2] B. Owen, W. Kamleh, D. B. Leinweber, S. Mahbub and B. Menadue, Correlation matrix methods for excited meson form factors in full QCD, PoS LATTICE 2012, 173 (2012).

[3] D. B. Leinweber, W. Melnitchouk, D. G. Richards, A. G. Williams and J. M. Zanotti, Baryon spectroscopy in lattice QCD, Lect. Notes Phys. 663, 71 (2005) [nucl-th/0406032].

[4] J. N. Hedditch, W. Kamleh, B. G. Lasscock, D. B. Leinweber, A. G. Williams and J. M. Zanotti, Pseudoscalar and vector meson form-factors from lattice QCD, Phys. Rev. D 75, 094504 (2007) [hep-lat/0703014 [HEP-LAT]].

[5] D. B. Leinweber, R. M. Woloshyn and T. Draper, Electromagnetic structure of octet baryons, Phys. Rev. D 43, 1659 (1991).

[6] D. B. Leinweber, T. Draper and R. M. Woloshyn, Decuplet baryon structure from lattice QCD, Phys. Rev. D 46, 3067 (1992) [hep-lat/9208025].

[7] S. Boinepalli, D. B. Leinweber, A. G. Williams, J. M. Zanotti and J. B. Zhang, Precision electromagnetic structure of octet baryons in the chiral regime, Phys. Rev. D 74, 093005 (2006) [hep-lat/0604022].

[8] M. S. Mahbub, A. O.Cais, W. Kamleh, B. G. Lasscock, D. B. Leinweber and A. G. Williams, Isolating Excited States of the Nucleon in Lattice QCD, Phys. Rev. D 80, 054507 (2009) [arXiv:0905.3616 [hep-lat]].

[9] M. S. Mahbub et al. [CSSM Lattice Collaboration], Roper Resonance in 2+1 Flavor QCD, Phys. Lett. B 707, 389 (2012) [arXiv:1011.5724 [hep-lat]].

[10] S. Aoki et al. [PACS-CS Collaboration], 2+1 Flavor Lattice QCD toward the Physical Point, Phys. Rev. D 79, 034503 (2009) [arXiv: 0807.1661 [hep-lat] ].

[11] M. G. Beckett, B. Joo, C. M. Maynard, D. Pleiter, O. Tatebe and T. Yoshie, Building the International Lattice Data Grid, Comput. Phys. Commun. 182, 1208 (2011) [arXiv : 0910.1692 [hep-lat] ].

[12] I. C. Cloet, D. B. Leinweber and A. W. Thomas, Delta baryon magnetic moments from lattice QCD, Phys. Lett. B 563, 157 (2003) [hep-lat/0302008].

[13] D. S. Roberts, W. Kamleh and D. B. Leinweber, Wave Function of the Roper from Lattice QCD, arXiv:1304.0325 [hep-lat].

[14] I. C. Cloet, D. B. Leinweber and A. W. Thomas, Simple quark model with chiral phenomenology, Phys. Rev. C 65, 062201 (2002) [hep-ph/0203023].

[15] M. S. Mahbub et al., Low-lying Odd-parity States of the Nucleon in Lattice QCD, Phys. Rev. D 87, 011501 (2013) [arXiv:1209.0240 [hep-lat]]. 